PII: S0045-6535(98)00042-3

\title{
COMPREHENSIVE APPROACH TO DETERMINING THE PHYSICAL PROPERTIES OF GRANULAR ACTIVATED CARBONS
}

\author{
YU-CHUN CHIANG ${ }^{1}$, PEN-CHI CHIANG ${ }^{1 *}$ AND E-E CHANG $^{2}$ \\ 1. Graduate Institute of Environmental Engineering, National Taiwan University, \\ 71 Chou-Shan Rd., Taipei, Taiwan, ROC. \\ 2. Department of Analytical Chemistry, Taipei Medical College, Taipei, Taiwan, ROC.
}

(Received in Germany 29 September 1997; accepted 9 January 1998)

\begin{abstract}
The objectives of this research were to identify the physical characteristics of activated carbons manufactured by peat, bituminous coal and coconut shell as well as examine the correlation among these properties. The helium density and mercury density were measured by using the recommended standard methods; in addition, the adsorption isotherms of nitrogen, argon and mercury were reported by using the automatic adsorption apparatus. The results manifested the pore features could be realized from the adsorption isotherm and hysteresis loop of nitrogen. In addition, as the percent of macropore volume was significant, the composite approach would be recommended as an alternative for analyzing pore features. The result of factor analysis implied the characteristic energy of adsorption was associated with the pore size distribution. It intended, except for physical adsorption, other mechanisms might coact on adsorption processes. O1998 Elsevier Science Ltd. All rights reserved
\end{abstract}

Keywords: Activated carbon, hysteresis loop, composite approach, pore size distribution, factor analysis

\section{INTRODUCTION}

Activated carbon adsorption has been considered as one of the promising technologies for controlling toxic and hazardous air pollutants and for recovering valuable chemicals at some specific industries. Activated carbons are the porous carbonaceous adsorbents; generally, they can be made of various raw materials such as peat, coal, nut-shell, lignite, saw dust and synthetic polymers. Two major forms, powder and granular, are very popular in commercial uses. For gas phase applications, cylindrically extruded pellets of 4-6 mm, or crushed and sieved granules of 4/8 mesh to $10 / 20$ mesh are commonly used [1]. In general, based on their different manufacture processes, activated carbons can be classified into 
two types, $\mathrm{H}$-carbon and L-carbon, which will cause different reactions during adsorption processes [2].

Identifying the surface microstructure of activated carbons is an essential procedure for design and use of activated carbon adsorption. Inert gas adsorption is one of the conventional technologies to determine their physical structure. According to BET classification, five physical adsorption isotherms can be identified; of which, Type I, IV and V isotherms represent the characteristic of porous solids [3]. Based on the classification suggested by International Union of Pure \& Applied Chemistry (IUPAC), adsorption on microporous $(<2 \mathrm{~nm})$ solids generates an isotherm of Type I; while a Type IV isotherm results from adsorption on solids with mesopores (from 2 to $50 \mathrm{~nm}$ ) and macropores (>50 nm) [4].

The most important application of Type IV isotherms is to determine the pore size distribution of the adsorbent. Kelvin addressed a cylindrical model to calculate the pore size [3]. Since the applicable range of Kelvin equation was limited to $P / P_{0}>0.42$, which has been approved to be the closure of hysteresis loop of nitrogen isotherms, Lippens \& de Boer [5] developed a t-method and then Mikhail et al. [6] extended the t-method to propose a micropore method (MP-method) for obtaining a reliable and complete distribution under low pressure conditions. Horvath \& Kawazoe [7] utilized an average potential function to develop a slit model for determining the pore size distribution in microporous carbons, but it could not predict reasonable values for larger pores. Recently, Saito \& Foley [8] compared the slit-pore model with the cylindrical-pore model and suggested that the cylindrical pore model was a more promising method for assessing the micropore size distribution because it took account of the effect of curvature and corresponded well with a number of physical parameters.

It is impressive that a Type IV isotherm usually possesses a hysteresis loop where, for any amount adsorbed, the value of $\mathrm{P} / \mathrm{P}_{\mathrm{o}}$ is always lower at the desorption stage than at the adsorption. The shape of hysteresis loop is not only determined by the shape of pores but also dependent on the pore size distribution. de Boer [9] first proposed a classification to distinguish five major types of hysteresis loops. Afterwards, the IUPAC recommended a new classification of hysteresis loops, which categorized loops into four groups. A Type H1 loop characterized the agglomerates of spheroidal particles having fairly uniform size, while corpuscular systems would generate a $\mathrm{H} 2$ loop. Types $\mathrm{H} 3$ and $\mathrm{H} 4$ existed in the isotherms of the samples with slit-shaped pores or plate-like particles [10].

A number of adsorption isotherms, such as Langmuir, BET, universal t-curve, Dubinin-Radushkevich (D-R) and Dubinin-Astakhov (D-A) methods, have been published in the literature. However, most of them were lack of physical significance owing to the fact that actual mechanisms between the adsorbent and the adsorbates have not yet been identified. The objectives of this research were to understand the surface structural characteristics of activated carbons, as well as to discuss the specific correlation among these properties by using the factor analysis. This research will do good for the development of substantial adsorption models in the future. 


\section{MATERIALS AND METHODS}

\section{Materials}

Eight kinds of commercial activated carbons were used in this study. P1-P4 were peat carbons with a pelleted form; $\mathrm{B} 1-\mathrm{B} 3$ were granular bituminous carbons; $\mathrm{C} 1$ was a granular coconut shell carbon. Wherein P1-P4 and B1 were obtained from Norit Co. (the Netherlands); B2-B3 were provided by Calgon Co. (America); $\mathrm{Cl}$ was purchased from Liangchien $\mathrm{Co}$. (Taiwan). The names and sizes of activated carbons could refer to Table 1 in detail. Two of them had special treatments: P3 was acid-washed and P4 was impregnated. All activated carbons were pretreated at oven of $250^{\circ} \mathrm{C}$ purged with pure nitrogen in vacuo overnight for removing the moisture and other contaminants prior to making the analyses below.

\section{Methods}

\section{Adsorption isotherms}

Adsorption isotherms were measured from ASAP 2000 system (accelerated surface area and porosimetry system, V2.02) [11]. Before the isotherms were reported, the activated carbon samples were outgassed at $250^{\circ} \mathrm{C}$ to clean the surface of the sample. Nitrogen was used as the degas backfill and analytical gas, while helium was used for the analysis of free space. It took about 7-12 $\mathrm{h}$ to complete an adsorption-desorption isotherm at $77 \mathrm{~K}$. The similar approach was also used for the analysis of micropores of activated carbons with the replacement of nitrogen by argon. Pressure points at the low end of the isotherms were obtained by using a $3.00 \mathrm{cc} \mathrm{g}^{-1}$ dose amount up to a relative pressure of 0.001 [12]. However, it needed spend about sixty hours finishing a complete analysis. Adsorption isotherms of mercury were obtained from Autopore II 9220 V1.05. The contact angle was 130 degrees and the mercury surface tension was $485 \mathrm{dyn} / \mathrm{cm}$. The range of applied pressure was from 0 to 55,000 psia [13].

\section{Density analysis [14] [15]}

The true density $\left(\rho_{H e}\right)$ of activated carbon was determined by gas pycnometer (Accupyc 1330, Micromeritics). Helium was used as the analytical gas with a pressure of 20 psia. A $10 \mathrm{~cm}^{3}$ activated carbon sample was weighted and purged 10 times with helium before measuring. Then the mean value of 10 runs for each sample was calculated. The apparent density $\left(\rho_{H z}\right)$ of activated carbon was analyzed by utilizing mercury intrusion technology, which was based on the assumption that mercury does not penetrate into the pores with a diameter less than $150,000 \stackrel{\circ}{A}$. The apparatus of this method was simple and easy to operate, but the result was slightly dependent on the shape of activated carbons. According to the measurements of true density and apparent density, the total pore volume and porosity could be determined from the appropriate algorithms. 


\section{Statistical analysis}

The specific relationship of the parameters correlated with the adsorptive capacity of activated carbon was examined by using the factor analysis of SAS. Factor analysis grouped a number of the properties of activated carbons into fewer categories according to the level of their specific correlation. Consequently, the major properties in a factor possessed the same pattern of variation or influence, where a special correlation was expected to be present. The modeling performance could be evaluated by the index of explained variance. In this study for a certain factor, a property which possessed an absolute value of the factor loading greater than 0.7 was regard as a striking property characterizing this factor. According to the characteristics of each factor, it rendered to infer some efficient and useful strategies to improve the specific adsorption capacity and put activated carbons to good uses.

\section{RESULTS AND DISCUSSION}

\section{Adsorption Isotherms}

Figure 1 illustrated the adsorption-desorption isotherms of nitrogen at $77 \mathrm{~K}$ on $\mathrm{P} 1, \mathrm{~B} 3$ and $\mathrm{C} 1$ carbons. According to the BET classification, all of the isotherms gave a form of Type IV, which clearly demonstrated that a hysteresis loop existed in each isotherm. In addition, the isotherms raised upward slightly when the pressure approached to the saturated pressure, which implied the occurrence of multilayer adsorption. The onset of the hysteresis loop in each isotherm approximately took place at a relative pressure of 0.4 , which meant the action of capillary condensation could start from the pore size about $30 \stackrel{\circ}{A}$. The shape of hysteresis loops for the bituminous carbons was significantly different from that found in other carbons. This might be attributed to its smaller particle sizes, where there were interstices of particles contributing rather additional adsorptive spaces. In addition, it seemed to suppose the pores were Type $\mathrm{H} 3$ or Type H4, i.e., the pores were slit-shaped capillaries or formed from the plate-like particles. All carbons had a significant "shoulder point", which indicated a rapid expulsion of adsorbate happened at the corresponded relative pressure [3].

The adsorption isotherms of argon at $77 \mathrm{~K}$ were also shown in Figure 1. It was presumed that the amounts of vapors similar in nature adsorbed on an adsorbent would come to an agreement when the saturated relative pressure was approached. However, it was only validated in B3 adsorption, where the limiting adsorption volume was about $400 \mathrm{cc} \mathrm{g}^{-1}$. There were quite different amounts between argon and nitrogen taken up by $\mathrm{P} 1$ and $\mathrm{C} 1$, which intended the assumption of liquid-like condensation within the pores was not suitable for these carbons. Meanwhile, the areas between the nitrogen and argon curves might indicate the contribution from micropores in which argon molecules could enter but nitrogen molecules were prohibited. 
P1

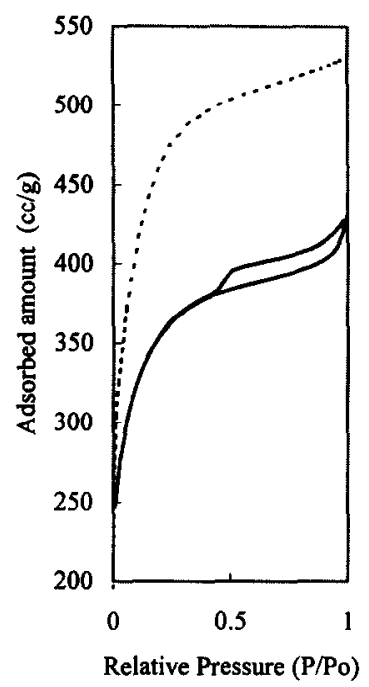

B3

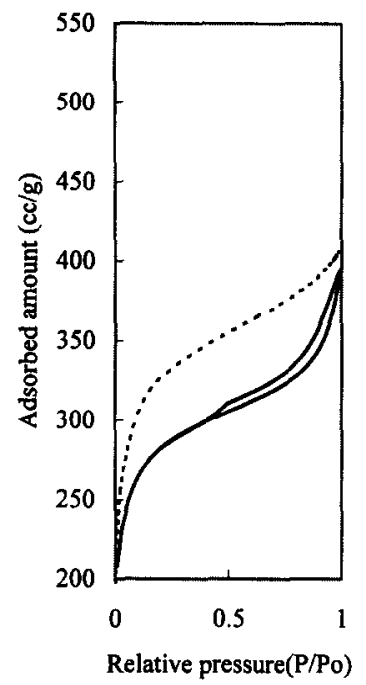

C1

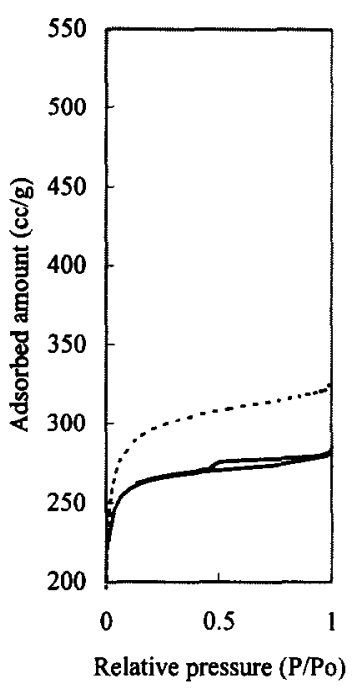

Figure 1. Adsorption isotherms of argon and nitrogen at $77 \mathrm{~K}$ on $\mathrm{P} 1, \mathrm{~B} 3$ and $\mathrm{C} 1$ carbons.

\section{Characteristics of Surface Area}

Table 1 shows the specific surface areas of activated carbons with the analysis of adsorption isotherms of argon at $77 \mathrm{~K}$ by using the BET and Langmuir equations. It was observed that as the data with relative pressures in the normal BET range, i.e., $0.05<P / P_{0}<0.35$, were used, the BET plots had a negative intercept, which accounted for the fact that the surface of activated carbon was homogeneous or approximately uniform [3]. As a result, a preferable top limit of relative pressures of 0.14 was suggested. The $c$ values of the BET equation for carbons studied were higher than 100 . Wherein the coconut carbon $(\mathrm{Cl})$, possessing only a specific surface area of $975 \mathrm{~m}^{2} \mathrm{~g}^{-1}$, had a high $\mathrm{c}$ value of 761 under relative pressures between 0.01 to 0.08 . This finding was responsible for the sharp knee in its isotherm and implied that there were considerable proportion of micropores in coconut-shelled carbons. The ratios of the specific surface area of BET equation to that of Langmuir equation for all carbons were also listed in Table 1, which indicated that the ratio for $\mathrm{B} 3$ was the highest, about 1.44; while the ratios for $\mathrm{P} 2$ and $\mathrm{C} 1$ were the lowest, only 1.23. Consequently, it could infer that $\mathrm{B} 3$ possessed a larger mean pore size yet $\mathrm{P} 2$ and $\mathrm{C} 1$ had lower ones.

\section{Characteristics of Pores}

\section{Density approach}

Table 2 summarizes the measurements of true density and apparent density of activated carbons. The apparent densities of the activated carbons ranged from 0.87 to $1.29 \mathrm{~g} \mathrm{cc}^{-1}$; in addition, the average true 
Table 1. Summary of the basic information and specific surface areas of activated carbons measured with argon adsorption isotherms at $77 \mathrm{~K}$ by using BET and Langmuir equations.

\begin{tabular}{|c|c|c|c|c|c|c|c|}
\hline \multirow{2}{*}{$\begin{array}{l}\text { Activated } \\
\text { carbon }\end{array}$} & \multirow[t]{2}{*}{ Name } & \multirow[t]{2}{*}{ Size } & \multicolumn{3}{|c|}{ BET method } & \multirow{2}{*}{$\begin{array}{c}\text { Langmuir } \\
\text { method } \\
\text { Area, } \mathrm{S}_{\mathrm{L}} \\
\left(\mathrm{m}^{2} / \mathrm{g}\right)\end{array}$} & \multirow[b]{2}{*}{$\mathbf{S}_{\mathbf{L}} / \mathbf{S}_{\mathbf{B}}$} \\
\hline & & & $\mathrm{P} / \mathrm{P}_{\mathrm{o}}$ range & $\begin{array}{c}\text { Area, } S_{B} \\
\left(\mathrm{~m}^{2} / \mathrm{g}\right)\end{array}$ & c & & \\
\hline P1 & Sorbonorit 3 & $3 \mathrm{~mm}$ & $0.05-0.14$ & 1472 & 126 & 2060 & 1.40 \\
\hline $\mathrm{P} 2$ & Norit RB 3 & $3 \mathrm{~mm}$ & $0.05-0.08$ & 1120 & 331 & 1373 & 1.23 \\
\hline P3 & Norit RX 3 extra & $3 \mathrm{~mm}$ & $0.05-0.12$ & 1315 & 141 & 1766 & 1.34 \\
\hline P4 & Norit RBAA 3 & $3 \mathrm{~mm}$ & $0.05-0.12$ & 877 & 141 & 1159 & 1.32 \\
\hline B 1 & Darco Vapure & $4 / 10$ & $0.05-0.08$ & 1120 & 331 & 1426 & 1.27 \\
\hline B2 & Filtrasorb 100 & $8 / 30$ & $0.05-0.08$ & 841 & 441 & 1196 & 1.42 \\
\hline B3 & Filtrasorb 400 & $12 / 40$ & $0.05-0.12$ & 1027 & 361 & 1483 & 1.44 \\
\hline $\mathrm{C} 1$ & Unicarb & $4 / 8$ & $0.01-0.08$ & 975 & 761 & 1196 & 1.23 \\
\hline
\end{tabular}

densities were between 2.06 to $2.27 \mathrm{~g} \mathrm{cc}^{-1}$. The calculation of total pore volume through the algorithm of true and apparent densities indicated P1 and P3 (peat) exhibited a porosity over $60 \%$; however, bituminous carbons with porosity of 40 to $45 \%$ possessed much lower pore volumes than the other carbons. This suggested that, compared to other carbons, the bituminous carbons should have a number of pores sizing on the macropore range, which would allow mercury to penetrate into the pores and result in overestimating the apparent density. Accordingly, the macropore volume of bituminous carbons should provide a striking capacity for adsorption.

\section{Composite approach}

Although the total pore volume could be obtained from the density approach, the detailed pore size distribution was still obscure. Due to the lack of available methods to determine the pore volume distribution for the whole range of relative pressures, a composite distribution curve was made up by combination of the Horvath \& Kawazoe (H-K) method, the Kelvin equation and the mercury porosimetry, which was suggested in the literature [6][7]. According to the IUPAC classification, the pore volumes of micropores, mesopores and macropores were shown in Figure 2 and their sums were listed in Table 2. It was impressive that the sums of micropore and mesopore volumes exceeded $95 \%$ at least for all carbons. In addition, B2 and B3 had lower percents of micropore volume, as well as had larger percents of macropore volume. Table 2 also indicated the mean pore size of the coconut carbon was the smallest, $13.94^{\circ} \mathrm{A}$; but $\mathrm{B} 2$ and B3 possessed higher ones, 17.51 and $17.94 \stackrel{\circ}{A}$, which might be associated with the amount of macropore volume. To sum up, the percents of micropore volume were between $57-85 \%$ for the activated 


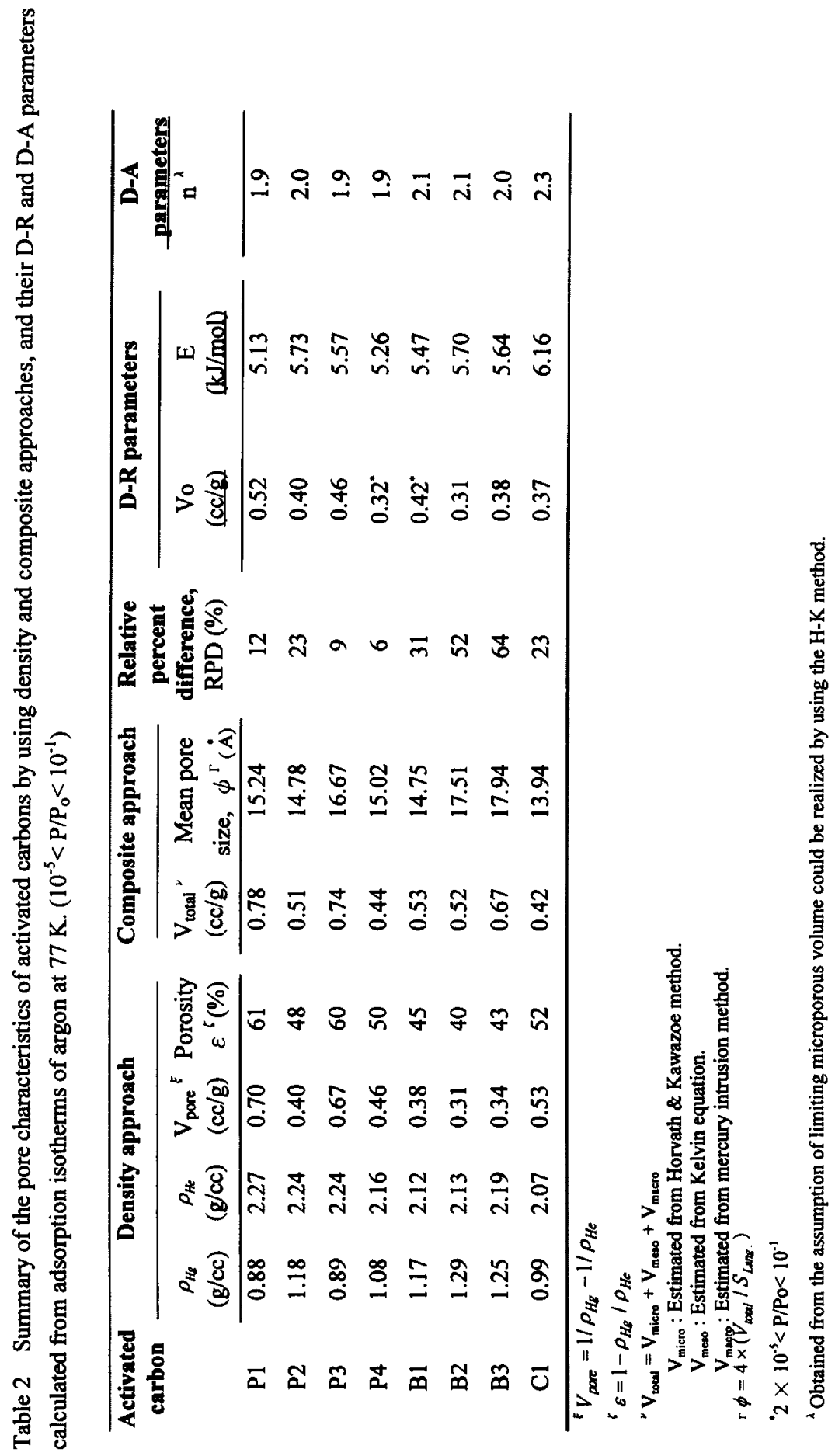




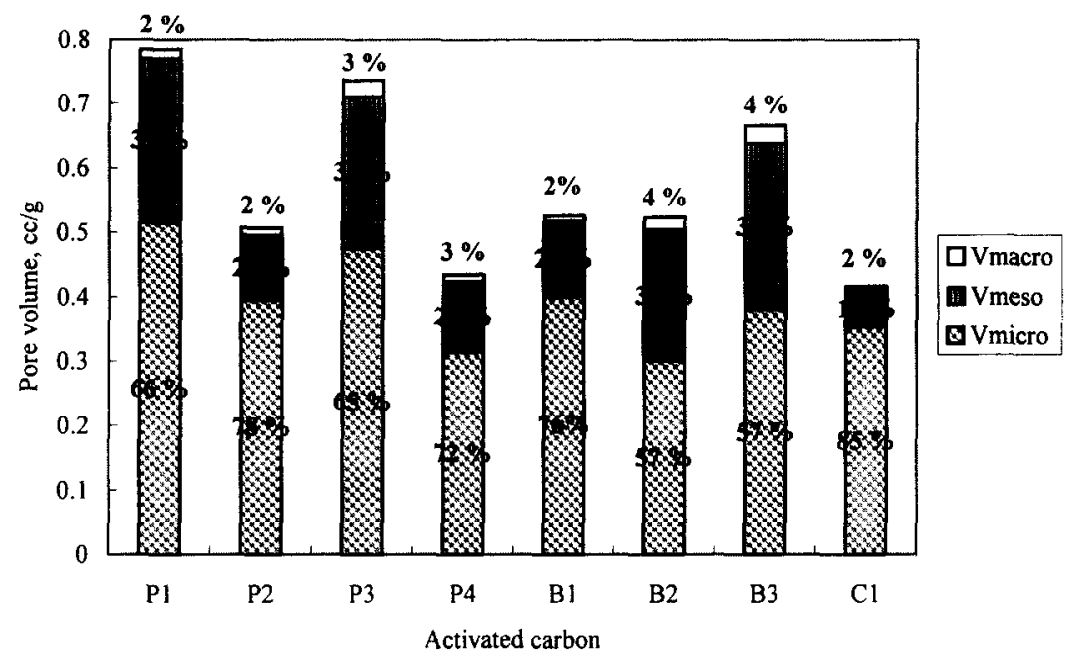

Figure 2. Pore volume distributions of activated carbons based on the IUPAC classification.

carbons studied. The density approach apparently underestimated the pore volumes for bituminous carbons, which was consistent with the previous discussion. The differences in the total pore volumes estimated from these two approaches were visualized by calculating their relative percent difference (RPD) as shown in Table 2. The RPD values of bituminous carbons, namely 31,52 , and 64 , were apparently higher than other carbons, which suggested that the total pore volume calculated from density approach would be underestimated because the mercury molecules intruded into the pore sizing in the macropore range.

\section{Micropore's features}

Since the amount of micropore volume was predominant from the previous discussion, the applications of Dubinin-Radushkevich (D-R) and Dubinin-Astakhov (D-A) equations were considered below. The estimates of parameters in equations were listed in Table 2, which were calculated by using the data of adsorption isotherms of argon at $77 \mathrm{~K}$ with a fit range of relative pressures from $10^{-5}$ to $10^{-1}$. It was found that the characteristic energies of adsorption (E) for all selected adsorbents in this study were over $5 \mathrm{~kJ} \mathrm{~mol}^{-1}$; among them, the coconut carbon had the highest value, $6.16 \mathrm{~kJ} \mathrm{~mol}^{-1}$. Using the micropore volume obtained from the H-K method as the limiting micropore volume, the exponent $\mathrm{n}$ value of D-A equation, related to the homogeneity of the pore system, was realized for each carbon, which ranged from 1.9 to 2.3 . It indicated that the coconut carbon ( $\mathrm{C} 1)$ with a pore volume distribution centered on the micropore range had a $\mathrm{n}$ value of 2.3 ; however, the broader pore volume distribution of P1 was responsible for a lower $\mathrm{n}$ value of 1.9 . 

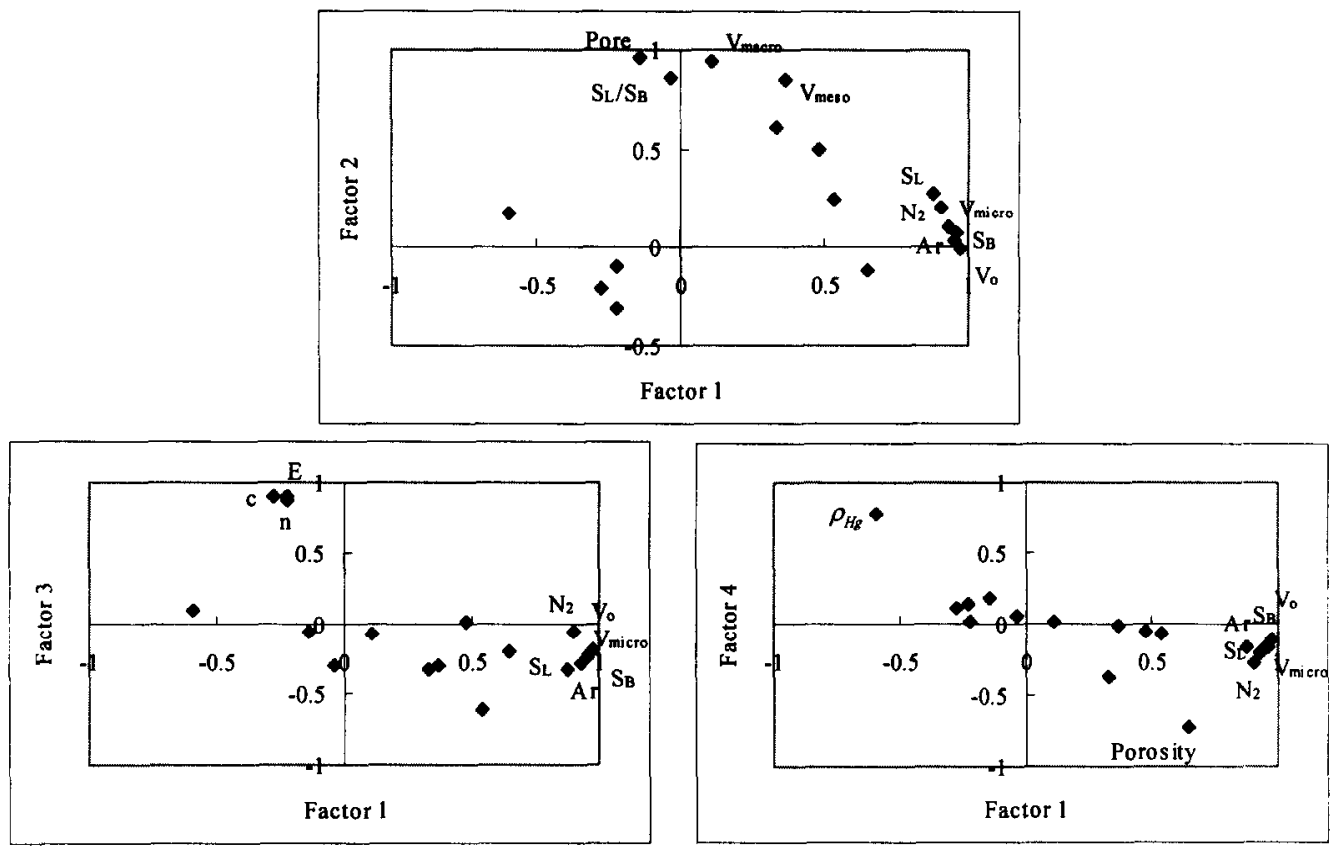

Figure 3. The result of factor analysis, where the major properties for each factor were marked.

\section{Classification of Structural Properties}

Figure 3 shows the result of factor analysis for the properties associated with adsorptive capacity of activated carbons. Four factors were retained with a explained variance of $90 \%$. The properties were pointed on each graph which represented a two-dimension constructed by two factors. In addition, for each factor the major parameters, possessed an absolute value of factor loading higher than 0.7 , were marked extraordinarily. Factor 1 grouped argon and nitrogen adsorbed amounts together with limit micropore volume $\left(\mathrm{V}_{0}\right)$, micropore volume $\left(\mathrm{V}_{\text {micro }}\right)$, BET surface area $\left(\mathrm{S}_{\mathrm{B}}\right)$ and Langmuir surface area $\left(\mathrm{S}_{\mathrm{L}}\right)$. This factor explained that the micropore volume and specific surface area were the primary properties for argon and nitrogen adsorption. The mean pore size, macropore volume $\left(V_{\text {macro }}\right)$, the ratio of $S_{L}$ to $S_{B}$ and mesopore volume $\left(\mathrm{V}_{\text {meso }}\right)$ were categorized in factor 2 . It indicated that the adsorbed layer on activated carbon depended on its pore structure, especially on macropores and mesopores. When the pore volume mostly contributed from macropores and mesopores, the mean pore size should be large as well as the adsorbed layer, i.e., $\mathrm{S}_{\mathrm{L}} / \mathrm{S}_{\mathrm{B}}$, should be too. On the other hand, it seemed to infer that the BET surface area might be suitable to describe the adsorptive capacity of activated carbon since the Langmuir surface area will be influenced by the pore features. Factor 3 pointed out a specific positive relationship existed in the characteristic energy of adsorption (E), $c$ value and $n$ value. This outcome suggested that except for physical mechanisms, other chemical interactions might happen during adsorption because $E$ was related to $\mathrm{n}$ which was a function concerning the adsorbent and the adsorbate. The last factor obviously implied that 
the overestimation of mercury density would result in the underestimation of porosity, which was responsible for the risk of the application of density approach. As a result, the composite approach should be recommended as a reliable method to examine the pore size distribution.

\section{CONCLUSIONS}

The findings suggested that the pores of activated carbons in this study were slit-shaped capillaries or resulted from the plate-like particles. It was apparent that the more the area of hysteresis loop, the more the mesopore volume. The best-fit range of relative pressures for the BET equation should be under 0.14 ; moreover, the Langmuir surface area would make more discrete from the BET surface area when pore sizes were larger. Since the macropores would suffer from the intrusion of mercury molecules, the density approach might underestimate the porosity of activated carbons. Therefore the composite approach would be recommended as an alternative. In addition, over $95 \%$ of pore volume was located in the ranges of micropores and mesopores for all studied carbons. It was approved that the peat carbons with a smaller $\mathbf{n}$ value of D-A equation had a broader pore size distribution; while the coconut carbon with the highest $n$ value possessed a narrower one. In conclusion, the surface area and micropore volume of activated carbons were mainly predominant their adsorptive capacities; nevertheless, this research also found out the characteristic energy of adsorption was related to the pore size distribution, which seemed to imply the surface chemistry would influence the adsorption processes. This hypothesis needs to be further studied and confirmed in our future research work.

\section{ACKNOWLEDGEMENTS}

The authors express their sincere thanks to the National Science Council, Taiwan, ROC for its financial support ( Contract no. NSC 86-2221-E-002-071) of this study.

\section{REFERENCES}

[1] M. Suzuki, Adsorption Engineering, Kodansha LTD., Tokyo (1990).

[2] M. O. Corapcioglu and C. P. Huang, The Surface Acidity and Characterization of Some Commercial Activated Carbons, Carbon, 25, 569-578 (1987).

[3] S. J. Gregg and K. S. W. Sing, Adsorption, Surface Area and Porosity, Academic Press, New York (1967).

[4] B. McEnaney, Adsorption and Structure in Microporous Carbons, Carbon, 26, 267-274 (1988). 
[5] B. C. Lippens and J. H. de Boer, Studies on Pore Systems in Catalysts V. The $\mathbf{t}$ Method, Journal of Catalysis, 4, 319-323 (1965).

[6] R. S. Mikhail, S. Brunauer and E. E. Bodor, Investigations of a Complete Pore Structure Analysis, Journal of Colloid and Interface Science, 26, 45-53 (1968).

[7] G. Horvath and K. Kawazoe, Method for The Calculation of Effective Pore Size Distribution in Molecular Sieve Carbon, Journal of Chemical Engineering of Japan, 16, 470-475 (1983).

[8] A. Saito and H. C. Foley, Curvature and Parametric Sensitivity in Models for Adsorption in Micropores, AIChE Journal, 37, 429-436 (1991).

[9] J. H. de Boer, The Structure and Properties of Porous Materials, Butterworth, London (1958).

[10] V. P. Zhdanov, Application of Percolation Theory to Describing Kinetic Processes in Porous Solids, Advances in Catalysis, 39, 1-50 (1993).

[11] ASAP 2000 Accelerated Surface Area and Porosimetry System, Operator's Manual, V2.02 (1992).

[12] Micropore Analysis using the ASAP 2000 Accelerated Surface Area and Porosimetry System, Operator's Manual, V3.00 (1993).

[13] A. W. Adamson, Physical Chemistry of Surfaces, fourth edition, John Wiley \& Sons, New York (1982)

[14] M. Smisek, Active Carbon: Manufacture, Properties and Applications, Elsevier Publishing Company, New York (1970).

[15] H. Jankowska, A. Swiatkowski and J. Choma, Active Carbon, Ellis Horwood, New York (1991). 